\title{
Twisted modular forms and parabolic cohomology
}

\author{
by \\ Min Ho LeE (Cedar Falls, IA)
}

1. Introduction. Twisted modular forms are obtained by essentially pulling the usual modular forms back via an equivariant holomorphic map of the Poincaré upper half plane into itself. Let $\mathcal{H}$ be the Poincaré upper half plane, and let $\Gamma \subset \mathrm{SL}(2, \mathbb{R})$ be a Fuchsian group of the first kind. We denote by $j: \mathrm{SL}(2, \mathbb{R}) \times \mathcal{H} \rightarrow \mathbb{C}$ the automorphy factor given by $j(\gamma, z)=c z+d$ for $z \in \mathcal{H}$ and $\gamma=\left(\begin{array}{ll}a & b \\ c & d\end{array}\right) \in \mathrm{SL}(2, \mathbb{R})$. Then the usual modular forms of weight $k$ for $\Gamma$ are defined by using the automorphy factor $j(\gamma, z)^{k}$ for $\gamma \in \Gamma$ and $z \in \mathcal{H}$. In order to consider twisted modular forms we need a holomorphic map $\omega: \mathcal{H} \rightarrow \mathcal{H}$ that is equivariant with respect to a homomorphism $\chi$ : $\Gamma \rightarrow \operatorname{SL}(2, \mathbb{R})$, which means that $\omega(\gamma z)=\chi(\gamma) \omega(z)$ for all $\gamma \in \Gamma$ and $z \in \mathcal{H}$. Then twisted modular forms of weight $k$ associated to $\Gamma, \omega$ and $\chi$ are defined by using the automorphy factor $j(\chi(\gamma), \omega(z))^{k}$ for $\gamma \in \Gamma$ and $z \in \mathcal{H}$.

As an example of an equivariant pair $(\omega, \chi)$ we can consider the period map $\omega$ of an elliptic surface $E$ over the Riemann surface $X=\Gamma \backslash \mathcal{H} \cup\{$ cusps $\}$ and the monodromy representation $\chi$ of $E$. There are also naturally defined holomorphic maps $\omega$ which are equivariant with respect to the monodromy representations $\chi$ of a certain class of second order linear ordinary differential equations defined on a Riemann surface. Twisted modular forms of weight three are closely related to the theory of elliptic surfaces. To be more specific, let $E_{0}$ be the generic fiber of an elliptic surface $E$ over $X$. Then $E_{0}$ is an elliptic curve over the function field $K(X)$ of $X$, and the Poincaré normal map associated to $E_{0}$ satisfies a certain second order linear ordinary differential equation. If $\omega$ is the quotient of two linearly independent homogeneous solutions of the given differential equation and if $\chi$ is the monodromy representation, then each solution of the differential equation determines a twisted modular form of weight three (see e.g. [2], [4], [6], [7], [16]). Twisted modular forms of higher weights were consid-

2000 Mathematics Subject Classification: 11F11, 11F75, 14C30.

Key words and phrases: twisted modular forms, parabolic cohomology, Eichler-Shimura isomorphism. 
ered by Stiller [17] in connection with such linear ordinary differential equations.

Let $f: \mathcal{H} \rightarrow \mathbb{C}$ be a usual elliptic cusp form of weight $m+2$ for $\Gamma$, and set

$$
a_{\nu}(\gamma)=\int_{z_{0}}^{\gamma z_{0}} f(z) z^{\nu} d z
$$

for $\nu=0,1, \ldots, m$, where $z_{0} \in \mathcal{H} \cup \mathbb{Q} \cup\{\infty\}$. Then the vector-valued function $\Phi_{f}: \Gamma \rightarrow \mathbb{C}^{m+1}$ given by

$$
\Phi_{f}(\gamma)={ }^{\mathrm{t}}\left(a_{0}(\gamma), a_{1}(\gamma), \ldots, a_{m}(\gamma)\right) \in \mathbb{C}^{m+1}
$$

for all $\gamma \in \Gamma$ is a 1-cocycle which determines an element of the parabolic cohomology $H_{P}^{1}\left(\Gamma, V^{m}\right)$ of $\Gamma$ with coefficients in $V^{m}$, where $V^{m}$ is the $m$ th symmetric power of $V=\mathbb{C}^{2}$ whose $\Gamma$-action is induced by the standard representation of $\operatorname{SL}(2, \mathbb{R})$ on $\mathbb{C}^{2}$. It is well known that the map $f \mapsto \Phi_{f}$ induces the Eichler-Shimura isomorphism of the form

$$
H_{P}^{1}\left(\Gamma, V^{m}\right)=S_{m+2}(\Gamma) \oplus \overline{S_{m+2}(\Gamma)},
$$

where $S_{m+2}(\Gamma)$ is the space of cusp forms of weight $m+2$ for $\Gamma$ (cf. [3], [15]). On the other hand, if $f$ is a twisted cusp form of weight $m+2$ associated to $\Gamma, \omega$ and $\chi$, then, by using the integrals

$$
\int_{z_{0}}^{\gamma z_{0}} f(z) \omega(z)^{\nu} d \omega(z)
$$

for $\nu=0,1, \ldots, m$, we obtain a 1 -cocycle in $H_{P}^{1}\left(\Gamma, V_{\chi}^{m}\right)$, where $V_{\chi}^{m}$ has the same underlying space as $V^{m}$ but its $\Gamma$-action is induced by the composition of $\chi$ and the standard representation of $\mathrm{SL}(2, \mathbb{R})$ on $\mathbb{C}^{2}$.

In this paper we obtain the analogue of the Eichler-Shimura isomorphism for twisted modular forms. We describe the isomorphism as a Hodge structure on the parabolic cohomology $H_{P}^{1}\left(\Gamma, V_{\chi}^{m}\right)$ using the methods of Bayer-Neukirch [1] and Zucker [18].

2. Twisted modular forms. Let $\mathcal{H}$ be the Poincaré upper half plane on which $\mathrm{SL}(2, \mathbb{R})$ acts by linear fractional transformations, and let $\Gamma \subset$ $\mathrm{SL}(2, \mathbb{R})$ be a Fuchsian group of the first kind. Let $\chi: \Gamma \rightarrow \mathrm{SL}(2, \mathbb{R})$ be a homomorphism, and let $\omega: \mathcal{H} \rightarrow \mathcal{H}$ be a holomorphic map that is equivariant with $\chi$, which means that

$$
\omega(\gamma z)=\chi(\gamma) \omega(z)
$$

for all $\gamma \in \Gamma$ and $z \in \mathcal{H}$. We assume that $\chi(\Gamma)$ is a Fuchsian group of the first kind and that the inverse image of a parabolic subgroup of $\Gamma^{\prime}=\chi(\Gamma)$ under $\chi$ is a parabolic subgroup of $\Gamma$ so that the $\Gamma$-cusps and $\Gamma^{\prime}$-cusps 
correspond. Given an element $\delta=\left(\begin{array}{ll}a & b \\ c & d\end{array}\right) \in \mathrm{SL}(2, \mathbb{R})$, we set

$$
j(\delta, w)=c w+d
$$

for all $w \in \mathcal{H}$.

DEFINITION 2.1. Let $k$ be a nonnegative integer. A holomorphic function $f: \mathcal{H} \rightarrow \mathbb{C}$ is said to be a twisted modular form of weight $k$ for $\Gamma$ associated to $\omega$ and $\chi$ if $f$ satisfies the following conditions:

(i) $f(\gamma z)=f(z) j(\chi(\gamma), \omega(z))^{k}$ for all $\gamma \in \Gamma$ and $z \in \mathcal{H}$.

(ii) $f$ is holomorphic at each $\Gamma$-cusp.

The function $f$ is said to be a twisted cusp form of weight $k$ for $\Gamma$ associated to $\omega$ and $\chi$ if (ii) is replaced with

(ii) ${ }^{\prime} f$ vanishes at each $\Gamma$-cusp.

We shall denote by $S_{k}(\Gamma, \omega, \chi)$ the space of twisted cusp forms of weight $k$ for $\Gamma$ associated to $\omega$ and $\chi$.

REMARK 2.2. If the condition (i) in Definition 2.1 is replaced with

$$
f(\gamma z)=f(z) j(\gamma, z)^{m} j(\chi(\gamma), \omega(z))^{k},
$$

then the given holomorphic function $f$ is said to be a mixed automorphic form of type $(m, k)$ associated to $\Gamma, \omega$ and $\chi$ (see [11]). Thus a twisted modular form of weight $k$ can be regarded as a mixed automorphic form of type $(0, k)$. Certain types of mixed automorphic forms arise naturally as holomorphic forms of the highest degree on some families of abelian varieties. For example, a mixed automorphic form of type $(2, k)$ can be identified with holomorphic forms of degree $k+1$ on the fiber product of $k$ copies of an elliptic surface. Various aspects of mixed automorphic forms have been investigated recently (see e.g. [8], [10]-[14]).

EXAMPLE 2.3. Let $\Gamma$ be a Fuchsian group of the first kind, and let $K(X)$ be the function field of the smooth complex algebraic curve $X=\Gamma \backslash \mathcal{H} \cup$ $\{$ cusps . Consider a second order linear differential equation

$$
\widetilde{\Lambda} \widetilde{f}=\left(\frac{d^{2}}{d x^{2}}+\widetilde{P}(x) \frac{d}{d x}+\widetilde{Q}(x)\right) \widetilde{f}=0
$$

for $x \in X$ and $\widetilde{P}(x), \widetilde{Q}(x) \in K(X)$ with regular singular points, whose singular points are contained in $\Gamma \backslash$ cusps $\} \subset X$. Let

$$
\Lambda f=\left(\frac{d^{2}}{d z^{2}}+P(z) \frac{d}{d z}+Q(z)\right) f=0
$$

for $z \in \mathcal{H}$ be the differential equation obtained by pulling the equation $\widetilde{\Lambda} \widetilde{f}=0$ via the natural projection map $\mathcal{H} \rightarrow \Gamma \backslash \mathcal{H} \subset X$. If $\omega_{1}$ and $\omega_{2}$ are linearly independent solutions of $\Lambda f=0$, then we denote by $S^{m}(\Lambda)$ the 
linear ordinary differential operator of order $m+1$ such that the $m+1$ functions

$$
\omega_{1}^{m}, \omega_{1}^{m-1} \omega_{2}, \ldots, \omega_{1} \omega_{2}^{m-1}, \omega_{2}^{m}
$$

are linearly independent solutions of the corresponding homogeneous equation

$$
S^{m}(\Lambda) f=0 .
$$

We set $\omega(z)=\omega_{1}(z) / \omega_{2}(z)$ for all $z \in \mathcal{H}$, and denote by $\chi: \Gamma \rightarrow \operatorname{SL}(2, \mathbb{R})$ the monodromy representation of $\Gamma$ for the second order equation $\Lambda f=0$. Given a function $\psi$ on $\mathcal{H}$, let $f^{\psi}$ be a solution of the equation $S^{m}\left(\Lambda^{2}\right) f=\psi$. Then the function

$$
\frac{d^{m+1}}{d \omega(z)^{m+1}}\left(\frac{f^{\psi}(z)}{\omega_{2}(z)^{m}}\right)
$$

is a twisted modular form of weight $m+2$ for $\Gamma$ associated to $\omega$ and $\chi$ (see [17, p. 32] for details).

3. Sheaves on Riemann surfaces. Let $\chi: \Gamma \rightarrow \operatorname{SL}(2, \mathbb{R})$ and $\omega$ : $\mathcal{H} \rightarrow \mathcal{H}$ be as in Section 2 satisfying $\omega(\gamma z)=\chi(\gamma) \omega(z)$ for all $\gamma \in \Gamma$ and $z \in \mathcal{H}$. We set $\Gamma^{\prime}=\chi(\Gamma)$, and denote by $\Sigma$ and $\Sigma^{\prime}$ the sets of $\Gamma$-cusps and $\Gamma^{\prime}$-cusps, respectively. Thus we can consider the associated Riemann surfaces

$$
X_{0}=\Gamma \backslash \mathcal{H}, \quad X_{0}^{\prime}=\Gamma^{\prime} \backslash \mathcal{H}, \quad X=\Gamma \backslash \mathcal{H} \cup \Sigma, \quad X^{\prime}=\Gamma^{\prime} \backslash \mathcal{H} \cup \Sigma^{\prime},
$$

where $X$ and $X^{\prime}$ are compact, and there are natural inclusion maps

$$
\eta: X_{0} \rightarrow X, \quad \eta^{\prime}: X_{0}^{\prime} \rightarrow X^{\prime} .
$$

The natural action of $\mathrm{SL}(2, \mathbb{R})$ on $\mathbb{C}^{2}$ induces a representation of $\Gamma^{\prime}$ on $\mathbb{C}^{2}$. Let $V$ be the complex vector space $\mathbb{C}^{2}$ regarded as a representation space of $\Gamma^{\prime}$, and for a nonnegative integer $m$ let $V^{m}$ be the $m$ th symmetric power of $V$. Let $\mathcal{O}_{\mathcal{H}}$ be the sheaf of holomorphic functions on $\mathcal{H}$, and set

$$
\mathcal{O}_{\mathcal{H}}\left(V^{m}\right)=\mathcal{O}_{\mathcal{H}} \otimes V^{m},
$$

where $V^{m}$ is considered as a constant sheaf on $\mathcal{H}$. The group $\Gamma^{\prime}$ acts on $\mathcal{O}_{\mathcal{H}}$ by $\gamma \cdot f(z)=f\left(\gamma^{-1} z\right)$ for $\gamma \in \Gamma^{\prime}$ and $f \in \mathcal{O}_{\mathcal{H}}$. This action and the natural action of $\Gamma^{\prime}$ on $V$ induces an action of $\Gamma^{\prime}$ on $\mathcal{O}_{\mathcal{H}}(V)=\mathcal{O}_{\mathcal{H}} \otimes V=\mathcal{O}_{\mathcal{H}} \otimes \mathbb{C}^{2}$ given by

$$
\left(\gamma \cdot\left(\begin{array}{l}
f_{1} \\
f_{2}
\end{array}\right)\right)(z)=\gamma\left(\begin{array}{l}
f_{1} \\
f_{2}
\end{array}\right)\left(\gamma^{-1} z\right)=\left(\begin{array}{c}
a f_{1}+b f_{2} \\
c f_{1}+d f_{2}
\end{array}\right)\left(\frac{d z-b}{-c z+a}\right)
$$

for all $\gamma=\left(\begin{array}{ll}a & b \\ c & d\end{array}\right) \in \Gamma^{\prime}$ and

$$
\left(\begin{array}{l}
f_{1} \\
f_{2}
\end{array}\right) \in \mathcal{O}_{\mathcal{H}}(V)=\mathcal{O}_{\mathcal{H}} \otimes \mathbb{C}^{2} .
$$


Let $\mathcal{E}_{\mathcal{H}}$ be the subsheaf of $\mathcal{O}_{\mathcal{H}}(V)$ generated by the global section $\left(\begin{array}{l}z \\ 1\end{array}\right)$, that is,

$$
\mathcal{E}_{\mathcal{H}}=\left\{f(z)\left(\begin{array}{l}
z \\
1
\end{array}\right) \mid f \in \mathcal{O}_{\mathcal{H}}\right\} .
$$

LEMMA 3.1. The sheaf $\mathcal{E}_{\mathcal{H}}$ is invariant under the action of $\Gamma^{\prime}$.

Proof. Let $f \in \mathcal{O}_{\mathcal{H}}$ and $\gamma=\left(\begin{array}{ll}a & b \\ c & d\end{array}\right) \in \Gamma^{\prime}$. Then we have

$$
\begin{aligned}
\gamma \cdot f(z)\left(\begin{array}{l}
z \\
1
\end{array}\right) & =f\left(\gamma^{-1} z\right)\left(\begin{array}{l}
a\left(\gamma^{-1} z\right)+b \\
c\left(\gamma^{-1} z\right)+d
\end{array}\right) \\
& =\left(c\left(\gamma^{-1} z\right)+d\right) f\left(\gamma^{-1} z\right)\left(\begin{array}{l}
z \\
1
\end{array}\right) \\
& =(-c z+a) f\left(\frac{d z-b}{-c z+a}\right)\left(\begin{array}{l}
z \\
1
\end{array}\right)
\end{aligned}
$$

for all $z \in \mathcal{H}$, and hence it follows that $\mathcal{E}_{\mathcal{H}}$ is $\Gamma^{\prime}$-invariant.

Given a positive integer $m$, let $\mathcal{E}_{\mathcal{H}}^{m} \subset \mathcal{O}_{\mathcal{H}}\left(V^{m}\right)$ be the $m$ th symmetric power of $\mathcal{E}_{\mathcal{H}}$. The action of $\Gamma^{\prime}$ on $\mathcal{O}_{\mathcal{H}}(V)$ induces $\Gamma^{\prime}$-actions on $\mathcal{O}_{\mathcal{H}}\left(V^{m}\right)$ and on $\mathcal{E}_{\mathcal{H}}^{m}$. Let $\mathcal{O}_{\mathcal{H}}\left(V^{m}\right)^{\Gamma^{\prime}}$ (resp. $\left(\mathcal{E}_{\mathcal{H}}^{m}\right)^{\Gamma^{\prime}}$ ) denote the subsheaf of $\mathcal{O}_{\mathcal{H}}\left(V^{m}\right)$ (resp. $\left.\mathcal{E}_{\mathcal{H}}^{m}\right)$ consisting of the elements that are fixed under the $\Gamma^{\prime}$-action. Then $\mathcal{O}_{\mathcal{H}}\left(V^{m}\right)^{\Gamma^{\prime}}$ and $\left(\mathcal{E}_{\mathcal{H}}^{m}\right)^{\Gamma^{\prime}}$ determine sheaves $\mathcal{O}_{X_{0}^{\prime}}\left(V^{m}\right)$ and $\mathcal{E}_{X_{0}^{\prime}}^{m}$, respectively, on $X_{0}^{\prime}$ with $\mathcal{E}_{X_{0}^{\prime}}^{m} \subset \mathcal{O}_{X_{0}^{\prime}}\left(V^{m}\right)$. Let $\Omega_{X_{0}^{\prime}}^{1}$ be the sheaf of holomorphic 1-forms on $X_{0}^{\prime}$, and let $\Omega_{(2)}^{1}\left(\operatorname{resp} . \mathcal{O}_{(2)}\left(V^{m}\right)\right)$ be the sheaf on $X^{\prime}$ whose sections are the sections of $\eta^{*} \Omega_{X_{0}^{\prime}}^{1}$ (resp. $\left.\eta^{*} \mathcal{O}_{X_{0}^{\prime}}\left(V^{m}\right)\right)$ that are square-integrable near the cusps of $\Gamma^{\prime}$. We denote by $\mathcal{E}_{(2)}^{m}$ the extension of $\mathcal{E}_{X_{0}^{\prime}}^{m}$ in $\mathcal{O}_{(2)}\left(V^{m}\right)$.

Proposition 3.2. Let $\omega^{*}\left(\Omega^{1} \otimes \mathcal{E}^{m}\right)_{(2)}$ be the inverse image of the sheaf $\left(\Omega^{1} \otimes \mathcal{E}^{m}\right)_{(2)}=\Omega_{(2)}^{1} \otimes \mathcal{E}_{(2)}^{m}$ via the map $\omega_{X}: X \rightarrow X^{\prime}$ induced by $\omega: \mathcal{H} \rightarrow \mathcal{H}$. Then there is a canonical isomorphism

$$
S_{m+2}(\Gamma, \omega, \chi) \cong H^{0}\left(X, \omega^{*}\left(\Omega^{1} \otimes \mathcal{E}^{m}\right)_{(2)}\right)
$$

between the space of twisted cusp forms of weight $m+2$ for $\Gamma$ associated to $\omega$ and $\chi$, and the space of sections of the sheaf $\omega^{*}\left(\Omega^{1} \otimes \mathcal{E}^{m}\right)_{(2)}$ on $X$.

Proof. As in the proof of Lemma 12.15 of [18] the space of usual modular forms of weight $m+2$ for $\Gamma^{\prime}$ is canonically isomorphic to the space $H^{0}\left(X^{\prime},\left(\Omega^{1} \otimes \mathcal{E}^{m}\right)_{(2)}\right)$ of sections of $\left(\Omega^{1} \otimes \mathcal{E}^{m}\right)_{(2)}$ on $X^{\prime}$. Let $f$ be an element of $H^{0}\left(X, \omega^{*}\left(\Omega^{1} \otimes \mathcal{E}^{m}\right)_{(2)}\right)$. Then there is a section $f^{\prime}$ of $\left(\Omega^{1} \otimes \mathcal{E}^{m}\right)_{(2)}$ such that $f=f^{\prime} \circ \omega$. Thus we have

$$
\begin{aligned}
f(\gamma z) & =f^{\prime}(\omega(\gamma z))=f^{\prime}(\chi(\gamma) \omega(z))=j(\chi(\gamma), \omega(z))^{m+2} f^{\prime}(\omega(z)) \\
& =j(\chi(\gamma), \omega(z))^{m+2} f(z)
\end{aligned}
$$


for all $\gamma \in \Gamma$ and $z \in \mathcal{H}$; hence $f$ satisfies the transformation formula in Definition 2.1(i) for twisted modular forms of weight $m+2$. Since $f^{\prime}$ is a cusp form and since the $\Gamma$-cusps and $\Gamma^{\prime}$-cusps correspond, $f$ is a twisted cusp form of weight $m+2$ for $\Gamma$ associated to $\omega$ and $\chi$.

The complex vector space $\mathbb{C}^{2}$ possesses not only the $\Gamma^{\prime}$-action described above (in which case we wrote $V$ for $\mathbb{C}^{2}$ ) but also the $\Gamma$-action that is obtained by the composition of the homomorphism $\chi: \Gamma \rightarrow \mathrm{SL}(2, \mathbb{R})$ with the natural representation of $\operatorname{SL}(2, \mathbb{R})$ on $\mathbb{C}^{2}$. We shall use $V_{\chi}$ to denote $\mathbb{C}^{2}$ when it is regarded as the representation space of $\Gamma$ with respect to this action. For each nonnegative integer $k$ let $V_{\chi}^{k}$ denote the $k$ th symmetric power of $V_{\chi}$. Thus $\Gamma$ acts on $V_{\chi}^{k}$ by the composition of $\chi$ and the canonical irreducible representation of $\mathrm{SL}(2, \mathbb{R})$ on the $k$ th symmetric power of $\mathbb{C}^{2}$.

For each $\Gamma$-cusp $s \in \Sigma$ we denote by $\Gamma_{s}$ the parabolic subgroup of $\Gamma$ fixing $s$. Then the parabolic cohomology of $\Gamma$ with coefficients in $V_{\chi}^{m}$ is given by

$$
H_{P}^{1}\left(\Gamma, V_{\chi}^{m}\right)=\bigcap_{s \in \Sigma} \operatorname{Ker}\left[H^{1}\left(\Gamma, V_{\chi}^{m}\right) \rightarrow H^{1}\left(\Gamma_{s}, V_{\chi}^{m}\right)\right]
$$

The group $\Gamma$ acts on $\mathcal{H} \times V_{\chi}^{m}$ by

$$
\gamma \cdot(z, v)=\left(\gamma z,\left(S^{m} \circ \chi\right) v\right)
$$

for all $\gamma \in \Gamma$ and $(z, v) \in \mathcal{H} \times V_{\chi}^{m}$, where $S^{m}$ is the $m$ th symmetric tensor representation of $\operatorname{SL}(2, \mathbb{R})$ on $V_{\chi}^{m}=\mathbb{C}^{m+1}$. Then the quotient

$$
\tilde{V}_{\chi}^{m}=\Gamma \backslash \mathcal{H} \times V_{\chi}^{m}
$$

with respect to this action is the locally constant system on $X_{0}$ associated to $V_{\chi}^{m}$. Let $\eta_{*} \widetilde{V}_{\chi}^{m}$ be the direct image sheaf on $X$ of $\widetilde{V}_{\chi}^{m}$ via the inclusion map $\eta: X_{0} \rightarrow X$.

Proposition 3.3. The parabolic cohomology $H_{P}^{1}\left(\Gamma, V_{\chi}^{m}\right)$ of $\Gamma$ with coefficients in $V_{\chi}^{m}$ is canonically isomorphic to the first cohomology $H^{1}\left(X, \eta_{*} \widetilde{V}_{\chi}^{m}\right)$ of $X$ with coefficients in the sheaf $\eta_{*} \widetilde{V}_{\chi}^{m}$ on $X$.

Proof. The proof of this when the homomorphism $\chi$ is the inclusion map is given in [18, Proposition 12.5]. Essentially the same proof can be used for general $\chi$ as is sketched below. The Leray spectral sequence for $\eta: X_{0} \rightarrow X$ determines the exact sequence

$$
0 \rightarrow H^{1}\left(X, \eta_{*} \widetilde{V}_{\chi}^{m}\right) \rightarrow H^{1}\left(X_{0}, \widetilde{V}_{\chi}^{m}\right) \stackrel{\varpi}{\rightarrow} H^{0}\left(X, R^{1} \eta_{*} \widetilde{V}_{\chi}^{m}\right)
$$

For each cusp $s \in \Sigma$ we consider a small punctured disc $\Delta_{s}$ around $s$. Then we have

$$
H^{0}\left(X, R^{1} \eta_{*} \widetilde{V}_{\chi}^{m}\right)=\bigoplus_{s \in \Sigma} H^{1}\left(\Delta_{s}, \widetilde{V}_{\chi}^{m}\right)
$$


and the map $\varpi$ is the direct sum of the restriction maps

$$
\varpi_{s}: H^{1}\left(X_{0}, \widetilde{V}_{\chi}^{m}\right) \rightarrow H^{1}\left(\Delta_{s}, \widetilde{V}_{\chi}^{m}\right) .
$$

Thus, using the relations

$$
\begin{gathered}
H^{1}\left(X_{0}, \widetilde{V}_{\chi}^{m}\right)=H^{1}\left(\Gamma, V_{\chi}^{m}\right), \\
H^{1}\left(\Delta_{s}, \widetilde{V}_{\chi}^{m}\right)=H^{1}\left(\Gamma_{s} \backslash \mathcal{H}, \widetilde{V}_{\chi}^{m}\right)=H^{1}\left(\Gamma_{s}, V_{\chi}^{m}\right),
\end{gathered}
$$

we see that $\varpi_{s}$ can be regarded as the natural map

$$
\varpi_{s}: H^{1}\left(\Gamma, V_{\chi}^{m}\right) \rightarrow H^{1}\left(\Gamma_{s}, V_{\chi}^{m}\right)
$$

with $H_{P}^{1}\left(\Gamma, V_{\chi}^{m}\right)=\bigcap_{s \in \Sigma} \operatorname{Ker} \varpi_{s}$. Therefore we obtain the desired isomorphism from this and the above exact sequence.

4. Hodge structures. Given a nonnegative integer $k$, let $\mathcal{E}_{\mathcal{H}}^{k} \subset \mathcal{O}_{\mathcal{H}}\left(V^{k}\right)$ be as in Section 3. Since the map $\omega: \mathcal{H} \rightarrow \mathcal{H}$ is equivariant with respect to the homomorphism $\chi: \Gamma \rightarrow \mathrm{SL}(2, \mathbb{R})$ and $\mathcal{E}_{\mathcal{H}}^{k}$ is invariant under $\Gamma^{\prime}=\chi(\Gamma)$, the inverse image $\omega^{*}\left(\mathcal{E}_{\mathcal{H}}^{k}\right)$ of $\mathcal{E}_{\mathcal{H}}^{k}$ via the map $\omega: \mathcal{H} \rightarrow \mathcal{H}$ is $\Gamma$-invariant. We set

$$
\mathcal{F}_{\mathcal{H}}^{k}=\omega^{*}\left(\mathcal{E}_{\mathcal{H}}^{k}\right) \otimes \mathcal{O}_{\mathcal{H}}\left(V_{\chi}^{m-k}\right)
$$

Then $\mathcal{F}_{\mathcal{H}}^{k}$ is also $\Gamma$-invariant, and we obtain a $\Gamma$-invariant filtration

$$
\mathcal{O}_{\mathcal{H}}\left(V_{\chi}^{m}\right)=\mathcal{F}_{\mathcal{H}}^{0} \supset \mathcal{F}_{\mathcal{H}}^{1} \supset \ldots \supset \mathcal{F}_{\mathcal{H}}^{m} \supset \mathcal{F}_{\mathcal{H}}^{m+1}=\{0\}
$$

of the sheaf $\mathcal{O}_{\mathcal{H}}\left(V_{\chi}^{m}\right)$ on $\mathcal{H}$. If $\mathcal{O}_{X_{0}}\left(V_{\chi}^{m}\right)$ and $\mathcal{F}^{k}$ are the sheaves on $X_{0}=$ $\Gamma \backslash \mathcal{H}$ induced by the $\Gamma$-fixed sheaves $\mathcal{O}_{\mathcal{H}}\left(V_{\chi}^{m}\right)^{\Gamma}$ and $\left(\mathcal{F}_{\mathcal{H}}^{k}\right)^{\Gamma}$, respectively, then we obtain a filtration $\left\{\mathcal{F}^{k}\right\}_{k=0}^{\infty}$ of the sheaf $\mathcal{O}_{X_{0}}\left(V_{\chi}^{m}\right)$ on $X_{0}=\Gamma \backslash \mathcal{H}$ with

$$
\mathcal{O}_{X_{0}}\left(V_{\chi}^{m}\right)=\mathcal{F}^{0} \supset \mathcal{F}^{1} \supset \ldots \supset \mathcal{F}^{m} \supset \mathcal{F}^{m+1}=\{0\} .
$$

Let $\Omega_{X_{0}^{\prime}}^{\bullet}$ be the holomorphic de Rham complex on $X_{0}^{\prime}=\Gamma^{\prime} \backslash \mathcal{H}$. Then the pullback $\omega^{*} \Omega_{X_{0}^{\prime}}^{\bullet}=\omega_{X_{0}}^{*} \Omega_{X_{0}^{\prime}}^{\bullet}$ of $\Omega_{X_{0}^{\prime}}^{\bullet}$ via the map $\omega_{X_{0}}: X_{0} \rightarrow X_{0}^{\prime}$ induced by $\omega: \mathcal{H} \rightarrow \mathcal{H}$ is a complex on $X_{0}$. We set

$$
\omega^{*} \Omega_{X_{0}^{\prime}}^{\bullet}\left(V_{\chi}^{m}\right)=\omega^{*} \Omega_{X_{0}^{\prime}}^{\bullet} \otimes \mathcal{O}_{X_{0}}\left(V_{\chi}^{m}\right)
$$

and denote by $\omega^{*} \Omega_{(2)}^{\bullet}\left(V_{\chi}^{m}\right)$ the complex of sheaves on $X$ whose sections are the sections of the complex $\eta_{*}\left(\omega^{*} \Omega_{X_{0}^{\prime}}^{\bullet}\right)\left(V_{\chi}^{m}\right)$ that are square-integrable near the $\Gamma$-cusps.

Proposition 4.1. The cohomology $H^{i}\left(X, \eta_{*} \widetilde{V}_{\chi}^{m}\right)$ of $X$ with coefficients in the sheaf $\eta_{*} \widetilde{V}_{\chi}^{m}$ is canonically isomorphic to the hypercohomology

$$
\mathbb{H}^{i}\left(X, \omega^{*} \Omega_{(2)}^{\bullet}\left(V_{\chi}^{m}\right)\right)
$$

of the complex $\omega^{*} \Omega_{(2)}^{\bullet}\left(V_{\chi}^{m}\right)$ for each $i$. 
Proof. Let $V^{m}$ be as in Section 2, and let $\widetilde{V}^{m}=\Gamma^{\prime} \backslash \mathcal{H} \times V^{m}$ be the associated locally constant system on $X_{0}^{\prime}$. If $\eta^{\prime}: X_{0}^{\prime} \rightarrow X^{\prime}$ is the natural inclusion map as before, then by [18, Theorem 4.8] there is a canonical isomorphism

$$
H^{i}\left(X^{\prime}, \eta_{*}^{\prime} \widetilde{V}^{m}\right) \cong \mathbb{H}^{i}\left(X^{\prime}, \Omega_{(2)}^{\bullet}\left(V^{m}\right)\right),
$$

where $\Omega_{(2)}^{\bullet}\left(V^{m}\right)$ is the complex of sheaves on $X^{\prime}$ whose sections are the sections of the complex $\eta_{*}^{\prime}\left(\Omega_{X_{0}^{\prime}}^{\bullet}\right)\left(V^{m}\right)$ that are square-integrable near the $\Gamma^{\prime}$-cusps. Now the proposition follows by pulling back the sheaves $\eta_{*}^{\prime} \widetilde{V}^{m}$ and $\Omega_{(2)}^{\bullet}\left(V^{m}\right)$ via the map $\omega_{X_{0}}: X_{0} \rightarrow X_{0}^{\prime}$ induced by $\omega: \mathcal{H} \rightarrow \mathcal{H}$.

Let $\mathcal{O}_{X}$ and $\mathcal{O}_{X^{\prime}}$ be the sheaf of holomorphic functions on $X$ and $X^{\prime}$, respectively, and let $\Omega_{X^{\prime}}^{1}\left(\log \Sigma^{\prime}\right)$ be the sheaf of meromorphic 1-forms on $X^{\prime}$ that are holomorphic on $X_{0}^{\prime}$ and have a pole of order at most one at the $\Gamma^{\prime}$-cusps. Then the map

$$
d \otimes 1: \mathcal{O}_{\mathcal{H}}\left(V_{\chi}^{m}\right) \rightarrow \Omega_{\mathcal{H}}^{1} \otimes \mathcal{O}_{\mathcal{H}}\left(V_{\chi}^{m}\right)
$$

of sheaves on $\mathcal{H}$ induces the map

$$
\nabla: \omega^{*} \mathcal{O}_{X^{\prime}}\left(V_{\chi}^{m}\right) \rightarrow \omega^{*} \Omega_{X^{\prime}}^{1}\left(\log \Sigma^{\prime}\right) \otimes \mathcal{O}_{X}\left(V_{\chi}^{m}\right)
$$

of sheaves on $X$; here $\omega^{*}(\cdot)$ denotes the inverse image of the sheaf $(\cdot)$ via the map $\omega_{X}: X \rightarrow X^{\prime}$, and $(\cdot)\left(V_{\chi}^{m}\right)=(\cdot) \otimes V_{\chi}^{m}$. Now we consider the complex $K^{\bullet}$, where $K^{0}=\omega^{*} \mathcal{O}_{X^{\prime}}\left(V_{\chi}^{m}\right), K^{1}$ is the image of the map $\nabla$, and $K^{j}=\{0\}$ for $j \geq 2$. Then $\omega^{*} \Omega_{(2)}^{\bullet}\left(V_{\chi}^{m}\right)$ is a subcomplex of $K^{\bullet}$. We set

$$
F^{k}\left(\omega^{*} \Omega_{(2)}^{r}\right)\left(V_{\chi}^{m}\right)=\omega^{*} \Omega_{(2)}^{r}\left(\mathcal{F}^{k-r}\right)_{(2)}
$$

for each $k$ and $r$. Then the filtration $\left\{\mathcal{F}^{k}\right\}_{k=0}^{\infty}$ of $\mathcal{O}_{X_{0}}\left(V_{\chi}^{m}\right)$ constructed above induces a filtration $\left\{F^{k}\left(\omega^{*} \Omega_{(2)}^{\bullet}\right)\left(V_{\chi}^{m}\right)\right\}$ of the complex $\left(\omega^{*} \Omega_{(2)}^{\bullet}\right)\left(V_{\chi}^{m}\right)$, which in turn induces a filtration $\left\{F^{k} K^{\bullet}\right\}$ of the complex $K^{\bullet}$.

Proposition 4.2. The inclusion $\omega^{*} \Omega_{(2)}^{\bullet}\left(V_{\chi}^{m}\right) \rightarrow K^{\bullet}$ of filtered complexes induces an isomorphism

$$
\mathbb{H}^{\bullet}\left(X, \Omega^{\bullet}\left(V_{\chi}^{m}\right)\right) \cong \mathbb{H}^{\bullet}\left(X, K^{\bullet}\right)
$$

on hypercohomology.

Proof. As in the proof of Proposition 4.1, by pulling back the complexes in [18, Proposition 9.1] via $\omega_{X}: X \rightarrow X^{\prime}$, we see that the inclusion $\omega^{*} \Omega_{(2)}^{\bullet}\left(V_{\chi}^{m}\right) \rightarrow K^{\bullet}$ is a quasi-isomorphism, that is, it induces an isomorphism on the respective cohomology sheaves. Thus the proposition follows from the lemma given in [5, p. 447].

Proposition 4.3. The graded complex $\operatorname{Gr}^{\bullet}\left(K^{\bullet}\right)$ with

$$
\operatorname{Gr}^{p}\left(K^{\bullet}\right)=F^{p} K^{\bullet} / F^{p+1} K^{\bullet}
$$


associated to the filtration $\left\{F^{k} K^{\bullet}\right\}$ of the complex $K^{\bullet}$ described above is acyclic except for $p=0, m+1$.

Proof. This follows by pulling back each complex $\operatorname{Gr}^{p}\left(K^{\bullet}\right)$ contained in the proof of [18, Lemma 12.14] via the map $\omega_{X}: X \rightarrow X^{\prime}$.

THEOREM 4.4. The parabolic cohomology $H_{P}^{1}\left(\Gamma, V_{\chi}^{m}\right)$ of the group $\Gamma$ has a polarized Hodge decomposition of type $\{(m+1,0),(0, m+1)\}$ of the form

$$
H_{P}^{1}\left(\Gamma, V_{\chi}^{m}\right) \cong S_{m+2}(\Gamma, \omega, \chi) \oplus \overline{S_{m+2}(\Gamma, \omega, \chi)} .
$$

Proof. The sheaf $\mathcal{O}_{X_{0}}\left(V_{\chi}^{m}\right)$ can be shown to possess a structure of a polarized variation of Hodge structure of weight $m$ using the construction of such a structure when $\chi$ is the identity described in $[18, \S 12]$. Thus by [18, Theorem 11.6], there is a Hodge structure of weight $m+1$ on

$$
H^{1}\left(X, \eta_{*} V_{\chi}^{m}\right) \cong \mathbb{H}^{1}\left(X, \omega^{*} \Omega_{(2)}^{\bullet}\left(V_{\chi}^{m}\right)\right)
$$

(see Proposition 4.1) whose Hodge filtration is induced by the filtration on the complex $\left(\omega^{*} \Omega_{(2)}^{\bullet}\right)\left(V_{\chi}^{m}\right)$. Therefore from Proposition 4.2 it follows that for each $p$ the $(p, m+1-p)$ component of $H^{1}\left(X, \eta_{*} V_{\chi}^{m}\right)$ is determined by the hypercohomology $\mathbb{H}^{1}\left(X, \operatorname{Gr}^{p}\left(K^{\bullet}\right)\right)$. Hence, using Proposition 4.3, we obtain

$$
H^{1}\left(X, \eta_{*} \widetilde{V}_{\chi}^{m}\right)=H^{m+1,0} \oplus H^{0, m+1}=H^{m+1,0} \oplus \overline{H^{m+1,0}} .
$$

Since $H_{P}^{1}\left(\Gamma, V_{\chi}^{m}\right)$ is isomorphic to $H^{1}\left(X, \eta_{*} \widetilde{V}_{\chi}^{m}\right)$ by Proposition 3.2 , it suffices to prove that $H^{m+1,0}$ is isomorphic to $S_{m+2}(\Gamma, \omega, \chi)$. Since the Hodge filtration

$$
\left\{F^{k} H^{1}\left(X, \eta_{*} V_{\chi}^{m}\right)\right\}
$$

of $H^{1}\left(X, \eta_{*} V_{\chi}^{m}\right)$ is induced by the filtration of $\Omega_{(2)}^{\bullet}\left(V_{\chi}^{m}\right)$, we have

$$
H^{m+1,0}=F^{m+1} H^{1}\left(X, \eta_{*} \widetilde{V}_{\chi}^{m}\right)=\mathbb{H}^{1}\left(X, F^{m+1}\left(\omega^{*} \Omega_{(2)}^{\bullet}\right)\left(V_{\chi}^{m}\right)\right) .
$$

However, we have

$$
\begin{aligned}
\mathbb{H}^{1}\left(X, F^{m+1}\left(\omega^{*} \Omega_{(2)}^{\bullet}\right)\left(V_{\chi}^{m}\right)\right) \\
\quad=H^{1}\left(X, F^{m+1}\left(\omega^{*} \Omega_{(2)}^{0}\right)\left(V_{\chi}^{m}\right)\right) \oplus H^{0}\left(X, F^{m+1}\left(\omega^{*} \Omega_{(2)}^{1}\right)\left(V_{\chi}^{m}\right)\right),
\end{aligned}
$$

where

$$
\begin{aligned}
& F^{m+1}\left(\omega^{*} \Omega_{(2)}^{0}\right)\left(V_{\chi}^{m}\right)=\omega^{*} \Omega_{(2)}^{0} \otimes\left(\mathcal{F}^{m+1}\right)_{(2)}=0 \\
& F^{m+1}\left(\omega^{*} \Omega_{(2)}^{1}\right)\left(V_{\chi}^{m}\right)=\omega^{*} \Omega_{(2)}^{1} \otimes\left(\mathcal{F}^{m}\right)_{(2)}=\omega^{*}\left(\Omega^{1} \otimes \mathcal{E}^{m}\right)_{(2)} .
\end{aligned}
$$

Here $\omega^{*}\left(\Omega^{1} \otimes \mathcal{E}^{m}\right)_{(2)}$ is the extension of $\omega^{*}\left(\Omega^{1} \otimes \mathcal{E}^{m}\right)$ in $\omega^{*} \Omega^{\bullet}\left(V_{\chi}^{m}\right)_{(2)}$. Thus, using Proposition 3.1, we obtain

$$
H^{m+1,0}=H^{0}\left(X, \omega^{*}\left(\Omega^{1} \otimes \mathcal{E}^{m}\right)_{(2)}\right) \cong S_{m+2}(\Gamma, \omega, \chi),
$$

and the theorem follows from this. 
Remark 4.5. When $m=1$ in Theorem 4.4, the Hodge structures of the parabolic cohomology $H_{P}^{1}\left(\Gamma, V_{\chi}\right)$ associated to elliptic surfaces were studied in [2] and [4]. In particular, given $\Gamma, \omega$ and $\chi$ determined by an elliptic surface, the dimension of the $(1,1)$-component in the Hodge decomposition of $H_{P}^{1}\left(\Gamma, V_{\chi}\right)$ was given in [2, Proposition 3.20] by a formula that depends on the types of singular fibers contained in the given elliptic surface. In our case, however, because of the assumption that the inverse image of the parabolic subgroups of $\Gamma^{\prime}$ are parabolic subgroups of $\Gamma$ the only singular fibers involved are of type $I_{b}$ in the sense of Kodaira (see [8, p. 56], [9]), assuming that $\Gamma, \omega$ and $\chi$ come from an elliptic surface. Thus Theorem 4.4 above, in particular, implies that the $(1,1)$-component of $H_{P}^{1}\left(\Gamma, V_{\chi}\right)$ is zero if the associated elliptic surface has singular fibers of type $I_{b}$ only.

\section{References}

[1] P. Bayer and J. Neukirch, On automorphic forms and Hodge theory, Math. Ann. 257 (1981), 135-155.

[2] D. Cox and S. Zucker, Intersection numbers of sections of elliptic surfaces, Invent. Math. 53 (1979), 1-44.

[3] M. Eichler, Eine Verallgemeinerung der Abelschen Integrals, Math. Z. 67 (1957), 267-298.

[4] Y. Endo, Parabolic cohomology and generalized cusp forms of weight three associated to Weierstrass equations over function fields, Ph.D. thesis, Temple University, 1985.

[5] P. Griffiths and J. Harris, Principles of Algebraic Geometry, Wiley, New York, 1978.

[6] W. Hoyt, On surfaces associated with an indefinite ternary lattice, Lecture Notes in Math. 1135, Springer, Heidelberg, 1985, 197-210.

[7] —, On twisted Legendre equations and Kummer surfaces, in: Proc. Sympos. Pure Math. 49, part 1, Amer. Math. Soc., Providence, RI, 1989, 695-707.

[8] B. Hunt and W. Meyer, Mixed automorphic forms and invariants of elliptic surfaces, Math. Ann. 271 (1985), 53-80.

[9] K. Kodaira, On compact analytic surfaces II, Ann. of Math. 77 (1963), 563-626.

[10] M. H. Lee, Mixed cusp forms and holomorphic forms on elliptic varieties, Pacific J. Math. 132 (1988), 363-370.

[11] —, Mixed cusp forms and Poincaré series, Rocky Mountain J. Math. 23 (1993), 1009-1022.

[12] —, Mixed automorphic forms on semisimple Lie groups, Illinois J. Math. 40 (1996), $464-478$.

[13] —, Mixed automorphic vector bundles on Shimura varieties, Pacific J. Math. 173 (1996), 105-126.

[14] - Mixed Hilbert modular forms and families of abelian varieties, Glasgow Math. J. 39 (1997), 131-140.

[15] G. Shimura, Sur les intégrales attachés aux formes automorphes, J. Math. Soc. Japan 11 (1959), 291-311.

[16] P. Stiller, Automorphic Forms and the Picard Number of an Elliptic Surface, Vieweg, Braunschweig, 1984.

[17] —, Special values of Dirichlet series, monodromy, and the periods of automorphic forms, Mem. Amer. Math. Soc. 299 (1984). 
[18] S. Zucker, Hodge theory with degenerating coefficients: $L^{2}$-cohomology in the Poincaré metric, Ann. of Math. 109 (1979), 415-476.

Department of Mathematics

University of Northern Iowa

Cedar Falls, IA 50614, U.S.A.

E-mail: lee@math.uni.edu

Received on 11.10.1999

and in revised form on 24.7.2002 